\title{
Erratum
}

\section{Erratum: Hurd et al., "Cannabis and the Developing Brain: Insights into Its Long-Lasting Effects”}

In the article "Cannabis and the Developing Brain: Insights into Its Long-Lasting Effects" by Yasmin L. Hurd, Olivier J. Manzoni, Mikhail V. Pletnikov, Francis S. Lee, Sagnik Bhattacharyya, and Miriam Melis, which appeared on pages $8250-8258$ of the October 16, 2019 issue, one of the references was incomplete. The reference to Frau et al. (2019) should appear as shown:

Frau R, Miczán V, Traccis F, Aroni S, Pongor CI, Saba P, Serra V, Sagheddu C, Fanni S, Congiu M, Devoto P, Cheer JF, Katona I, Melis M (2019) Prenatal THC exposure produces a hyperdopaminergic phenotype rescued by pregnenolone. Nat Neurosci, in press. DOI: 10.1038/s41593-019-0512-2.

This error does not affect the conclusions of the article. The article has been updated online.

DOI: 10.1523/JNEUROSCI.2716-19.2019

\section{Erratum: Litvina et al., "BRAIN Initiative: Cutting-Edge Tools and Resources for the Community"}

In the article "BRAIN Initiative: Cutting-Edge Tools and Resources for the Community", by Elizabeth Litvina, Amy Adams, Alison Barth, Marcel Bruchez, James Carson, Jason E. Chung, Kristin B. Dupre, Loren M. Frank, Kathleen M. Gates, Kristen M. Harris, Hannah Joo, Jeff William Lichtman, Khara M. Ramos, Terrence Sejnowski, James S. Trimmer, Samantha White, and Walter Koroshetz, which appeared on pages 8275-8284 of the October 16, 2019 issue, incorrect numbers of NIH budget support for small businesses were included within the "Small business programs for novel tools and technologies in neuroscience research" section. On page 8276, "A specific percentage of the NIH extramural budget supports Small Business Innovation Research (SBIR) and Small Business Technology Transfer (STTR) grant awards, as required by law 3.2 and 3.65\%, respectively." should instead read, "A specific percentage of the NIH extramural budget supports Small Business Innovation Research (SBIR) and Small Business Technology Transfer (STTR) grant awards, 3.2 and $0.45 \%$ respectively (3.65\% total for Small Business programs as required by law).” This error does not affect the conclusions of the article, and the article has been corrected online. 